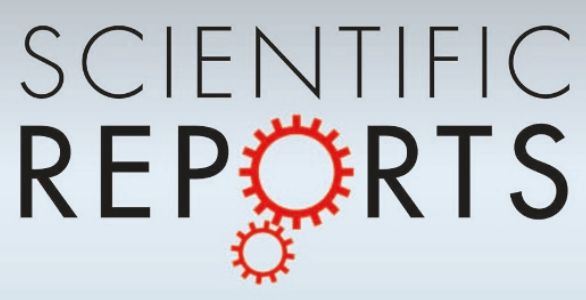

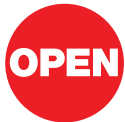

SUBJECT AREAS:

X-RAYS

FUEL CELLS

SCANNING PROBE

MICROSCOPY

PHASE-CONTRAST MICROSCOPY

Received

24 October 2012

Accepted

22 January 2013

Published

19 February 2013

Correspondence and requests for materials should be addressed to H.Y. (hyan@bnl.gov)

* Current address: Department of Physics,

University of Ulsan, Ulsan 680-749,

Korea.

\title{
Quantitative x-ray phase imaging at the nanoscale by multilayer Laue lenses
}

\author{
Hanfei Yan', Yong S. Chu' , Jörg Maser ${ }^{2,3}$, Evgeny Nazaretski' ', Jungdae Kim ${ }^{1 *}$, Hyon Chol Kang ${ }^{4}$, \\ Jeffrey J. Lombardo ${ }^{5} \&$ Wilson K. S. Chiu ${ }^{5}$ \\ ${ }^{1}$ National Synchrotron Light Source II, Brookhaven National Laboratory, Upton, NY 11 1973, USA, ${ }^{2}$ Advanced Photon Source, \\ Argonne National Laboratory, Argonne, Illinois 60439, USA, ${ }^{3}$ Center for Nanoscale Materials, Argonne, Illinois 60439, USA, \\ ${ }^{4}$ Department of Advanced Materials Engineering and BK21 Education Center of Mould Technology for Advanced Materials and \\ Parts, Chosun University, Gwangju 501-759, Republic of Korea, ${ }^{5}$ Department of Mechanical Engineering, University of \\ Connecticut, Storrs, Connecticut 06269-3139, USA.
}

For scanning $\mathrm{x}$-ray microscopy, many attempts have been made to image the phase contrast based on a concept of the beam being deflected by a specimen, the so-called differential phase contrast imaging (DPC). Despite the successful demonstration in a number of representative cases at moderate spatial resolutions, these methods suffer from various limitations that preclude applications of DPC for ultra-high spatial resolution imaging, where the emerging wave field from the focusing optic tends to be significantly more complicated. In this work, we propose a highly robust and generic approach based on a Fourier-shift fitting process and demonstrate quantitative phase imaging of a solid oxide fuel cell (SOFC) anode by multilayer Laue lenses (MLLs). The high sensitivity of the phase to structural and compositional variations makes our technique extremely powerful in correlating the electrode performance with its buried nanoscale interfacial structures that may be invisible to the absorption and fluorescence contrasts.

ecent progress in the development of $\mathrm{x}$-ray focusing optics has pushed the frontier of hard $\mathrm{x}$-ray nanofocusing into a regime well below $30 \mathrm{~nm}$, providing high focusing efficiencies even for hard $\mathrm{x}$-rays with photon energy above $10 \mathrm{keV}^{1,2}$. Technological advances in optics fabrication offer a unique opportunity for $\mathrm{x}$-ray imaging with high spatial resolution and allow to benefit from x-ray's excellent penetration power, high sensitivity to structural, elemental and chemical properties of a specimen, and insensitivity to external electromagnetic fields. We have pursued the development of MLL optics as a route towards nanometer scale hard x-ray imaging, and have built a scanning microscope using MLL optics ${ }^{3-8}$. Fluorescence imaging with a $2 \mathrm{D}$ resolution of $25 \times 27 \mathrm{~nm}^{2}$ has been demonstrated at the energy of $12 \mathrm{keV}$, and comparable performance was achieved at $19.5 \mathrm{keV}^{8}$. This emerging new capability enables studies of nanostructures with unprecedented spatial resolution by fully exploiting various contrast mechanisms for quantitative analysis.

In addition to the conventional absorption and fluorescence contrasts, it has been demonstrated that the differential phase contrast can be obtained in the scanning mode by analyzing the transmitted diffraction pattern in the far-field ${ }^{9-13}$. Unlike other phase imaging techniques ${ }^{14-19}$, DPC neither requires a fully coherent beam nor suffers from a phase wrapping problem, because it measures the phase gradient. On the other hand, its resolution is limited to the probe size. Different methods utilizing Wiener filter ${ }^{10}$, differential intensity ${ }^{9}$ and moment analysis ${ }^{13}$ have been developed to retrieve the phase quantitatively. Other methods based on wedged absorber ${ }^{12}$ or pinhole ${ }^{11}$ are mostly variants of the differential intensity method. The Wiener filter method assumes a weak phase object and is not valid for a thick specimen, which is often a case for real samples. The differential intensity method assumes a uniform exit wave field or pupil function for the focusing optic. The moment analysis method measures the convolution of the phase gradient with the $\mathrm{x}$-ray probe, and artifacts can be introduced if the probe function cannot be described by a simple model. To enable quantitative phase imaging capability for MLL optics and to take advantage of its high spatial resolution, we propose a highly robust and generic DPC algorithm that seeks a best-fit solution to the measured far-field intensity. Our method neither requires prior knowledge of a specimen nor the probe, allowing a maximum generality.

\section{Results}

Concept description. A schematic drawing in Fig. 1 depicts the experiment setup, and a detailed description is given in the Methods section. At the far-field detector plane, the Fraunhoffer diffraction condition is satisfied, and 


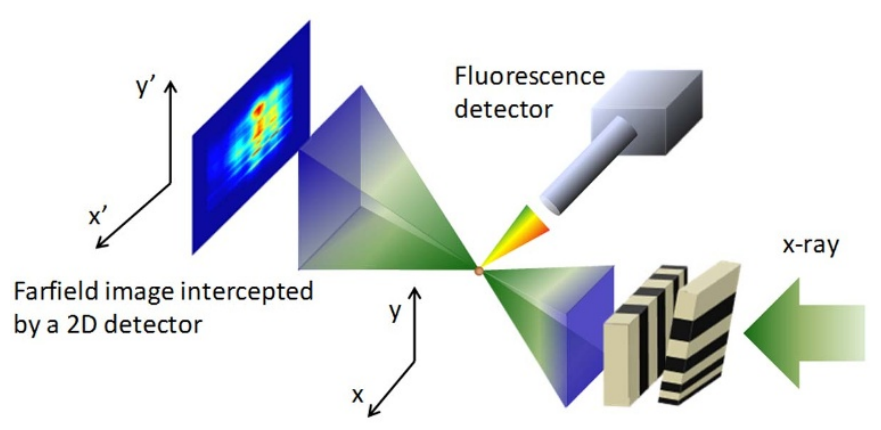

A pair of MLLs for nanofocusing

Figure $1 \mid$ Schematic drawing of the experimental setup. An incident plane wave is focused to a spot by two crossed MLLs. The fluorescence signal and the far-field diffraction pattern are recorded simultaneously as the specimen is raster-scanned.

the wave field arriving at the detector is related to that at the focal plane by a Fourier transform. We assume that the complex wave field at the focal plane is simply a product of the probe function, $P(\mathbf{r})$, and a specimen transmission function, $T\left(\mathbf{r}-\mathbf{r}_{l, m}\right)$, where $\mathbf{r}_{l, m}$ is the translation vector in a $2 \mathrm{D}$ scan. For a nanoprobe, the value of $P$ is appreciable only within a small area. We can therefore expand the amplitude, $a$, and phase $\phi$, of the transmission function into a Taylor series up to the first order about $\mathbf{r}$,

$$
T\left(\mathbf{r}-\mathbf{r}_{l, m}\right) \approx\left[a_{l, m}+o(\mathbf{r})\right] \exp \left[i \phi\left(-\mathbf{r}_{l, m}\right)+i \mathbf{g}_{l, m} \cdot \mathbf{r}+o\left(\mathbf{r}^{2}\right)\right] .
$$

Due to the property of Fourier transform, the resulting diffraction pattern in the far-field will be displaced and attenuated as compared with the case when the specimen is not present, corresponding to $T(\mathbf{r})=1$. The displacement of the diffraction pattern is proportional to the local phase gradient vector $\mathbf{g}_{l, m}$ and the attenuation is determined by $a_{l, m}$. The absorption gradient contribution is very small and can be neglected ${ }^{9}$. For equation (1) to be valid, the beam size must be small as compared to the characteristic length of the local phase variation; locally the specimen can be approximated to a prism with a linear phase gradient. Once the phase gradient vector is found the phase can be reconstructed by integration.

As mentioned earlier, the significant dynamical diffraction effect of MLL optics introduces a very nonuniform far-field diffraction pattern and a probe profile that cannot be approximated well to a delta function (see Figures S1 and S2 in supplementary materials). As a result a correct quantification of the phase gradient with existing methods becomes very difficult. To tackle this difficulty, we employ a non-linear fitting algorithm to seek a solution of the type shown in equation (1) that will give a least-square error to the measured farfield data. If the far-field wave field from a nanoprobe passing through a specimen only experiences attenuation and a shift due to the phase gradient, according to the Parseval's equation, a leastsquare fit of these two parameters can be found equivalently after an inverse Fourier transform of the diffraction pattern,

$$
R_{l, m}=\iint\left|\mathcal{F}^{-1}\left[I_{l, m}\right]-a_{l, m}^{2} \mathcal{F}^{-1}\left[I_{R}\right] \exp \left(i \mathbf{g}_{l, m} \cdot \mathbf{r}\right)\right|^{2} d^{2} \mathbf{r},
$$

where $R_{l, m}$ is the residue error, $I_{R}$ is the reference far-field diffraction intensity distribution in the absence of specimen, and $\mathcal{F}^{-1}$ is inverse Fourier transform operator. The Fourier-shift fitting process not only ensures a minimum error caused by noises but also removes contributions from high order phase terms by allowing a residue error. Because fitting is done in Fourier space, a sub-pixel intensity variation can be measured accurately, leading to high detection sensitivity. In the described method, only the shift of the diffraction pattern (not the pattern itself) is of interest. Thus a coherent beam is not required. A detailed derivation of the algorithm can be found in the Methods section.

Phase imaging of a SOFC anode. To verify the validity and demonstrate the advantages of our method, we investigated a SOFC anode sample, composed of Nickel (Ni) and Yttria-stablized Zirconia (YSZ) cermet ${ }^{20}$. The scanning electron microscope (SEM) image of the sample is shown in Fig. 2a. Figs. $2 \mathbf{b}-2 \mathbf{d}$ show the horizontal phase-gradient maps of the specimen obtained by the differential intensity, the moment analysis and Fourier-shift fitting algorithms, respectively. As it is evident from the comparison, the former two suffer from strong artifacts, resulting in false phase gradient distribution in the center and blurred contrast over the entire specimen. The poor image quality prevents using them for a quantitative phase reconstruction. In contrast, the Fourier-shift fitting algorithm gives superior contrast with no obvious artifacts.

Fig. 3a-3b show $\mathrm{Ni}$ and $\mathrm{Pt}^{21}$ fluorescence images, and Fig. $3 c-3 d x-$ ray transmission image and reconstructed phase image of the specimen. The fluorescence signal from the YSZ electrolyte suffered significant absorption and was too weak to be used for quantitative analysis ${ }^{22}$. The absorption and phase measure the integral change of the imaginary and real components of the refractive index along the beam direction through the specimen, therefore allowing the detection of the buried interfacial structures. Both of them reveal the isolated and connected pores under the surface in the $\mathrm{Ni}$ anode and YSZ electrolyte, which cannot be seen in the SEM image. The phase image displays even higher levels of structural details due to a higher phase contrast, capturing all the structural features both in the $\mathrm{Ni}$ anode and the YSZ electrolyte. For example, a crack on the top of the sample, indicated by an arrow in Fig. $3 \mathbf{d}$, can be clearly seen in the phase image but is barely detectable in both the absorption and the fluorescence images.

Composition imaging via absorption and phase measurements. Owing to the robustness and high detection sensitivity of our
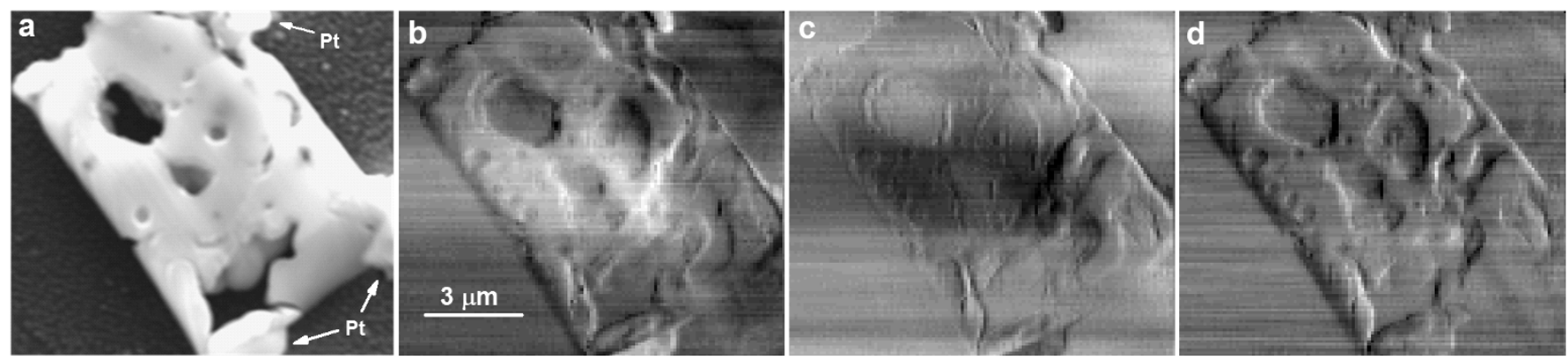

Figure $2 \mid$ (a) SEM image of the SOFC specimen adhered on a $\mathrm{Si}_{3} \mathrm{Ni}_{4}$ window with Pt welding. (b-d) are horizontal phase-gradient images obtained by differential intensity, moment analysis and Fourier-shift fitting algorithms, respectively. Artifacts and blurring effects can be seen in (b) and (c), as compared to (d). 

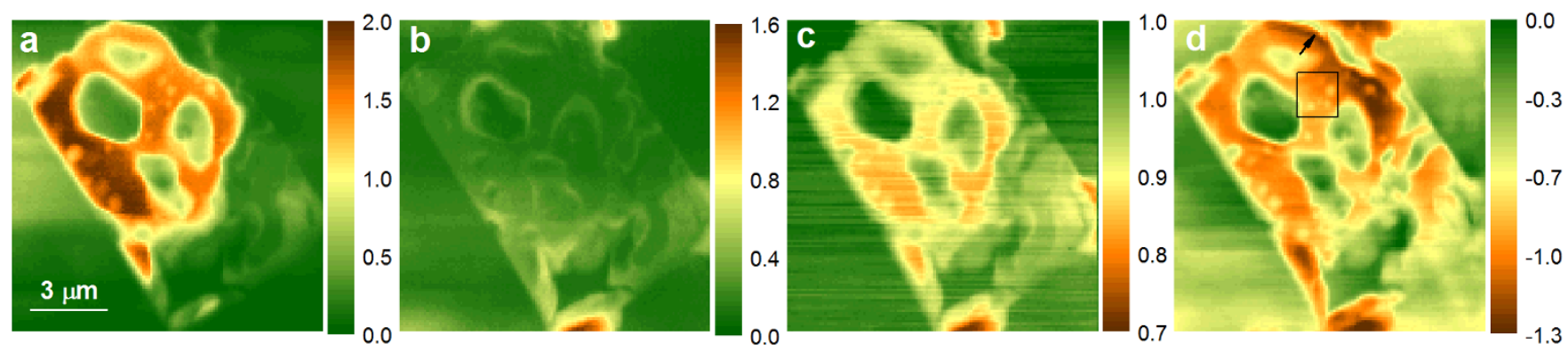

Figure $3 \mid \mathrm{Ni}$ K $\alpha$ fluorescence (a), Pt L $\alpha$ fluorescence (b), x-ray transmission (c) and reconstructed phase (d) images (units in radian) of the SOFC sample shown in Fig. 2a. The arrow in (d) points to a crack, which is barely seen in (a), (b) and (c). A zoom-in image of the rectangle area in (d) with a high resolution can be found in the supplementary material.

method, we were able to determine the refractive index decrement, $\delta$, and the absorption index, $\beta$, of the specimen with sufficiently high accuracy and consequently, able to exploit $\beta / \delta$ values as the imaging contrast. At fixed photon energy, this ratio provides unique fingerprint of the constituent elements, largely independent of the specimen thickness or the porosity. The composition image in Fig 4a, constructed from $\beta / \delta$ values, exhibits excellent correlation with the $\mathrm{Ni}$ and Pt fluorescence map. For a quantitative analysis, we plot the $\beta / \delta$ value along the yellow horizontal line in Fig. $4 \mathrm{~b}$. Comparison with the values for bulk $\mathrm{Ni}$ and YSZ indicates that the region from $\mathrm{x}$ $\approx-1.5$ through $\mathrm{x} \approx 3$ is occupied by $\mathrm{Ni}$ and $\mathrm{YSZ}$ phases and the region afterward predominantly by YSZ phase. To verify the consistency of the data, we introduce a constant scaling factor to correlate the thickness of $\mathrm{Ni}$ phase to its fluorescence intensity. Provided that the total thickness of the specimen is known, the composition can be calculated as well from the Ni fluorescence data. The constant scaling factor is determined by minimizing the error between composition values obtained from absorption and phase (shown as circles in Fig. 4b) and fluorescence (shown as black line in Fig. 4b). If all measured data contained no error, the two curves should coincide. The observed discrepancy is ascribed to the noisy absorption data, in particular in weak absorption region, and an oversimplified model for fluorescence. Nevertheless, the overall agreement is better than $30 \%$, which is very reasonable. This allows us to estimate the fraction of YSZ within the specimen in confidence (blue curve in Fig. 4b).

\section{Discussion}

Linking the micro/nano-structure evolution with SOFC's performance is a critical, yet unsolved, problem due to the lack of quantitative characterization tools with sufficient elemental, structural and chemical sensitivity at the nanoscale $e^{23,24}$. In particular for the detection of YSZ electrolyte, quantitative analysis using $\mathrm{x}$-ray fluorescence mapping remains challenging. The robust yet generic DPC analysis algorithm based on a Fourier-shift fitting process developed in this letter enable us to use MLL opitcs to perform parallel measurements of fluorescence, absorption and phase with spatial resolution better than $50 \mathrm{~nm}$ on a SOFC anode. The quantitative phase measurement leads to a new composition analysis technique that is complimentary to the conventional fluorescence analysis. Because of its generality, our DPC algorithm will benefit microscopy systems based on other nanofocusing optics which also produce complicated pupil function $^{25}$, and significantly advance the phase imaging capability for scanning x-ray microscopy.

\section{Methods}

Experiment setup. The experiment was conducted at 26-ID of Advanced Photon Source (APS) at Argonne National Laboratory. A monochromatic $\mathrm{x}$-ray beam with photon energy of $12 \mathrm{keV}$ was focused to a $\sim 30 \times 52 \mathrm{~nm}^{2}$ area by a pair of MLL optics placed orthogonally with respect to each other (see Figure $S 1$ in supplementary material for the focus profiles). A set of MLLs with apertures of $13.5 \mathrm{um}$ and 43.5 um were used to achieve focused flux of $\sim 4 \times 10^{7}$ photon/second. An order-sorting-aperture (OSA) was not used because the focus spot was separated from the direct beam ${ }^{8}$. An energydispersive silicon drift detector (Ketek, $30 \mathrm{~mm}^{2}$ active area) placed at about $90^{\circ}$ to the incident beam, was used to collect the $\mathrm{x}$-ray fluorescence signal emitted by a specimen positioned at the focal plane. The $\mathrm{x}$-rays transmitted through the specimen were captured by a $2 \mathrm{D}$ pixel-array detector (Pilatus $100 \mathrm{k}, 487 \times 195$ pixels, $172 \times 172 \mu \mathrm{m}^{2}$ pixel size) positioned about $1.4 \mathrm{~m}$ downstream from the specimen.

Sample preparation. The Ni/YSZ sample was fabricated using focused ion beam (FIB) milling ${ }^{26}$ and mounted on the silicon nitride window using a modification of the commonly used "lift out" procedure ${ }^{27,28}$. The sample has a plate shape with dimensions of $12 \times 8 \times 1.5 \mu^{3}$. First a section of a Ni/YSZ anode was mounted onto an SEM stand using carbon tape and loaded into the FIB/SEM vacuum chamber. Next, two staircase like trenches were milled into the bulk sample using the ion beam at $30 \mathrm{keV}$ with a current of $21 \mathrm{nA}$, on either side of a thin strip of the bulk material that is left intact. Once the craters were milled to a depth of 10 microns, the thin strip of Ni/YSZ was then thinned to 1.5 microns beginning with a current of $2.8 \mathrm{nA}$ at $30 \mathrm{keV}$, and then using progressively lower beam currents for the final surface cleaning. Following this thinning, a micromanipulator was attached to the top of the thinned sample using ion beam assisted Pt deposition. Next, the thinned sample was detached from the bulk material, and was moved to the silicon nitride window. Using the ion and electron SEM images, the sample was rotated and moved near the silicon nitride window until one corner of the sample was just touching the window. Pt was then deposited on the corner to lightly attach the sample to the window. The micromanipulator was then detached from the sample, and was used to push over the $\mathrm{Ni} / Y S Z$ until it was flat on the window. Finally, Pt was deposited on additional corners to hold the sample in place.

Image analysis algorithm. Assuming that the complex transmission function of the specimen is $T(\mathbf{r})$, we have,

$$
T(\mathbf{r})=\exp \left[i k \int_{0}^{t} n(\mathbf{r}, z) d z\right], \mathbf{r}=(x, y), k=2 \pi / \lambda, n=1-\delta+i \beta,
$$

where $t$ is the thickness, $\lambda$ is the wavelength and $n$ is the complex refractive index. Provided that Fraunhofer diffraction condition is satisfied, the complex wave field at the detector plane at each scanned point, $D_{l, m}$, can be related to the wave field at the focal plane by a Fourier transform,

$$
\begin{aligned}
D_{l, m}\left(\mathbf{k}^{\prime}\right) & =\frac{A}{2 \pi} \int_{-\infty}^{\infty} \int_{-\infty}^{\infty} P(\mathbf{r}) T\left(\mathbf{r}-\mathbf{r}_{l . m}\right) \exp \left(-i \mathbf{k}^{\prime} \cdot \mathbf{r}\right) d^{2} \mathbf{r}=A \mathcal{F}\left[P(\mathbf{r}) T\left(\mathbf{r}-\mathbf{r}_{l, m}\right)\right], \\
\mathbf{k}^{\prime} & =\frac{2 \pi}{\lambda L} \mathbf{r}^{\prime}, l=1,2, \ldots, N, m=1,2, \ldots, M .
\end{aligned}
$$

Here $A$ is a complex number contains all constants of no importance for this study, $L$ is the distance from the focal plane to the detector and $\mathbf{r}^{\prime}$ is the coordinates at the detector plane. For a small beam size, the following Taylor expansion is valid,

$$
\begin{aligned}
T\left(\mathbf{r}-\mathbf{r}_{l, m}\right) \approx\left[a_{l, m}+o(\mathbf{r})\right] \exp \left[i \phi\left(-\mathbf{r}_{l, m}\right)+i \mathbf{g}_{l, m} \cdot \mathbf{r}+\left(\mathbf{r}^{2}\right)\right], \\
a_{l, m}=a\left(-\mathbf{r}_{l, m}\right), \mathbf{g}_{l, m}=\nabla \phi\left(-\mathbf{r}_{l, m}\right),
\end{aligned}
$$

where both $a$ and $\phi$ are real functions. According to Fourier transform theorem, we have,

$$
I_{l, m}\left(\mathbf{k}^{\prime}\right)=a_{l, m}^{2} I_{R}\left(\mathbf{k}^{\prime}-\mathbf{g}_{l, m}\right), I_{l, m}=\left|D_{l, m}\right|^{2}, I_{R}=|A \mathcal{F}[P(\mathbf{r})]|^{2},
$$

where $I_{R}$ is the far-field diffraction intensity distribution in the absence of specimen.

For a measured 2D diffraction pattern $I_{l, m}$, we seek a vector $\mathbf{g}_{l, m}$ and a constant $a_{l, m}$ which will give the least-square error,

$$
R_{l, m}=\iint\left|I_{l, m}\left(\mathbf{k}^{\prime}\right)-a_{l, m}^{2} I_{R}\left(\mathbf{k}^{\prime}-\mathbf{g}_{l, m}\right)\right|^{2} d^{2} \mathbf{k}^{\prime}
$$

Based on the Parseval's equation, the integral is the same if we apply an inverse Fourier transform on the right-hand side of the equation, 

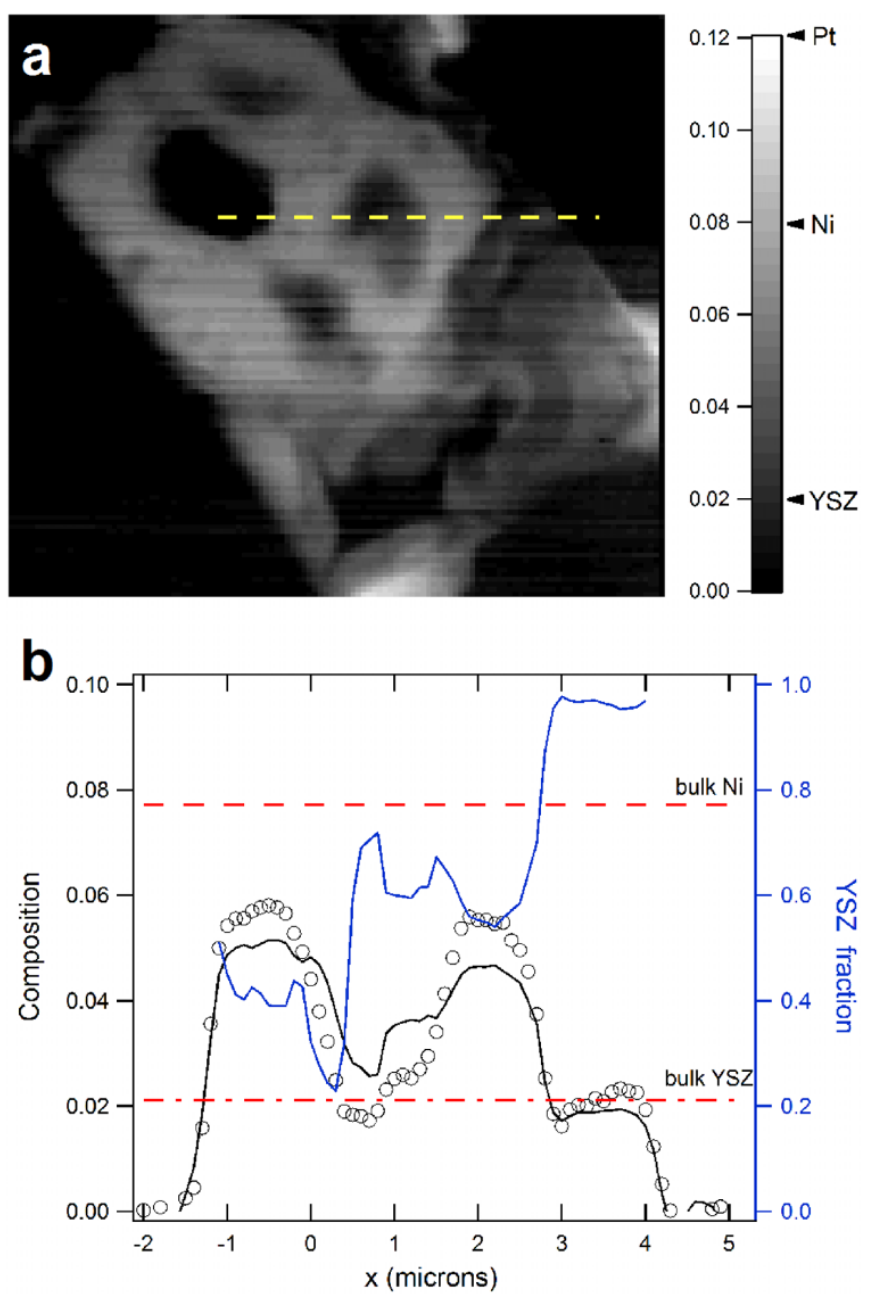

Figure $4 \mid$ (a) is the composition map displays the variation of the $\beta / \delta$ ratio. The bright region correlates well to the Pt- and Ni-rich areas.

The darker region in the bottom-right quadrant is mostly occupied by YSZ. A line plot across the yellow line is shown in (b) (black circle). The two red dashed lines correspond to the $\beta / \delta$ ratio for pure Ni (upper) and YSZ (lower) phases. The black solid curve is the $\beta / \delta$ ratio calculated from the $\mathrm{Ni}$ fluorescence data (see text for explanation). The two curves agree reasonably well, showing the consistency of the data. The blue line in (b) is the YSZ mole fraction extracted from the measurement.

$$
R_{l, m}=\iint\left|\mathcal{F}^{-1}\left[I_{l, m}\left(\mathbf{k}^{\prime}\right)\right]-a_{l, m}^{2} \mathcal{F}^{-1}\left[I_{R}\left(\mathbf{k}^{\prime}\right)\right] \exp \left(i \mathbf{g}_{l, m} \cdot \mathbf{r}\right)\right|^{2} d^{2} \mathbf{r} .
$$

Then a nonlinear fitting algorithm is employed to find the best estimates of $a_{l, m}$ and $\mathbf{g}_{l, m}$ which will give a least-square error to the measured far-field data ${ }^{29}$, provided that the transmission function can be approximated to the simple expression shown in equation (5). The residue error, $R_{l, m}$, also has a physical meaning. It contains not only background noise but also the deviation from the approximation shown in equation (5). If, for example, high order phase terms in equation (5) become more appreciable, a larger residue error is expected. Therefore, a map of the residue error indicates how dramatically the phase of the transmission function deviates from a linear variation in the range defined by the size of the focused beam. It also serves as a quantity indicating the validity of the linear approximation. Such maps are shown in Fig. S4 in supplementary materials.

In order to find a phase solution that will give a best estimate to the measured phase gradient data, we consider a continuous function consisting of a Fourier series,

$$
\phi(\mathbf{r})=\sum_{p=0}^{N-1} \sum_{q=0}^{M-1} c_{p, q} \exp \left(i \boldsymbol{\kappa}_{p, q} \cdot \mathbf{r}\right), \mathbf{\kappa}_{p, q}=2 \pi\left(\frac{q}{M \Delta x}, \frac{p}{N \Delta y}\right) .
$$

Here $\Delta x$ and $\Delta y$ are the scan step sizes in each direction. Again we seek a solution for the complex coefficients, $c_{l, q}$, which will give a least-square error,

$$
S=\sum_{l} \sum_{m}\left\{\left[\frac{\partial}{\partial x} \phi\left(\mathbf{r}_{l, m}\right)-g_{l, m}^{x}\right]^{2}+w\left[\frac{\partial}{\partial y} \phi\left(\mathbf{r}_{l, m}\right)-g_{l, m}^{y}\right]^{2}\right\}, w=\sigma_{x}^{2} / \sigma_{y}^{2} .
$$

Here $\sigma_{x}$ and $\sigma_{y}$ are the RMS noise of the phase gradient in each direction and $w$ is the weight. According to the differential property of Fourier transform and the Parseval's equation, one has,

$$
S=\sum_{p} \sum_{q}\left\{\left|i \kappa_{p, q}^{x} c_{p, q}-\tau_{p, q}^{x}\right|^{2}+w\left|i \kappa_{p, q}^{y} c_{p, q}-\tau_{p, q}^{y}\right|^{2}\right\}, \tau=\mathcal{F}[\mathbf{g}] .
$$

The superscript corresponds to x or y direction. Differentiating $S$ with respect to the real and imaginary component of each coefficient and equating them to zero, we arrive at,

$$
c_{p, q}=-i \frac{\kappa_{p, q}^{x} \tau_{p, q}^{x}+w \kappa_{p, q}^{y} \tau_{p, q}^{y}}{\left(\kappa_{p, q}^{x}\right)^{2}+w\left(\kappa_{p, q}^{y}\right)^{2}} .
$$

The coefficient for the zero frequency is determined from a known phase value at a reference point. One can prove that the commonly used Fourier integration $\operatorname{method}^{30}$ is equivalent to the special case here with $w$ equal to 1 .

1. Ice, G. E., Budai, J. D. \& Pang, J. W. L. The Race to X-ray Microbeam and Nanobeam Science. Science 334, 1234-1239, doi:10.1126/science.1202366 (2011).

2. Sakdinawat, A. \& Attwood, D. Nanoscale X-ray imaging. Nat. Photonics 4, 840-848, doi:10.1038/nphoton.2010.267 (2010).

3. Kang, H. C. et al. Nanometer Linear Focusing of Hard X Rays by a Multilayer Laue Lens. Physical Review Letters 96, 127401-127404 (2006).

4. Kang, H. C. et al. Sectioning of multilayers to make a multilayer Laue lens. Review of Scientific Instruments 78, 046103-046103 (2007).

5. Kang, H. C. et al. Focusing of hard $\mathrm{x}$-rays to 16 nanometers with a multilayer Laue lens. Applied Physics Letters 92, 221114 (2008).

6. Yan, H. F. X-ray dynamical diffraction from multilayer Laue lenses with rough interfaces. Physical Review B 79, 12, 165410 (2009).

7. Yan, H. F. et al. Takagi-Taupin description of x-ray dynamical diffraction from diffractive optics with large numerical aperture. Physical Review B 76, 115438 (2007).

8. Yan, H. F. et al. Two dimensional hard $\mathrm{x}$-ray nanofocusing with crossed multilayer Laue lenses. Optics Express 19, 15069-15076 (2011).

9. de Jonge, M. D. et al. Quantitative Phase Imaging with a Scanning Transmission X-Ray Microscope. Physical Review Letters 100, 163902 (2008).

10. Hornberger, B., Feser, M. \& Jacobsen, C. Quantitative amplitude and phase contrast imaging in a scanning transmission X-ray microscope. Ultramicroscopy 107, 644-655 (2007).

11. Kaulich, B. et al. Diffracting aperture based differential phase contrast for scanning X-ray microscopy. Optics Express 10, 1111-1117 (2002).

12. Mukaide, T., Takada, K., Watanabe, M., Noma, T. \& Iida, A. Scanning hard x-ray differential phase contrast imaging with a double wedge absorber. Review of Scientific Instruments 80, 033707 (2009).

13. Thibault, P. et al. Contrast mechanisms in scanning transmission x-ray microscopy. Physical Review A 80, 043813 (2009).

14. McNulty, I. et al. High-Resolution Imaging by Fourier Transform X-ray Holography. Science 256, 1009-1012 (1992)

15. Miao, J., Charalambous, P., Kirz, J. \& Sayre, D. Extending the methodology of Xray crystallography to allow imaging of micrometre-sized non-crystalline specimens. Nature 400, 342-344 (1999).

16. Thibault, P. et al. High-Resolution Scanning X-ray Diffraction Microscopy Science 321, 379-382 (2008).

17. Williams, G. J. et al. Fresnel Coherent Diffractive Imaging. Physical Review Letters 97, 025506-025504 (2006).

18. Chapman, H. N. \& Nugent, K. A. Coherent lensless X-ray imaging. Nat. Photon. 4, 833-839 (2010)

19. Abbey, B. et al. Keyhole coherent diffractive imaging. Nat. Phys. 4, 394-398 (2008).

20. Grew, K. N. et al. Nondestructive Nanoscale 3D Elemental Mapping and Analysis of a Solid Oxide Fuel Cell Anode. J. Electrochem. Soc. 157, B783-B792 (2010)

21. Pt is not an intrinsic element in this particular SOFC system but was extraneously introduced, predominantly near the corners, during the FIB-Pt bonding process.

22. The emission energies of the L-edge fluorescence (La1, La2, and Lb1) for $\mathrm{Zr}$ and $\mathrm{Y}$ range from 1.920 to $2.042 \mathrm{keV}$. Attenuation of these low energy fluorescence $\mathrm{x}$-rays through $1 \mathrm{um}$-thick $\mathrm{Ni}$ is about $17 \%$. For our experiment setting, where the fluorescence detector was placed at $90^{\circ}$ from the incidence beam as typical for scanning $\mathrm{x}$-ray microscopy setup, the fluorescence $\mathrm{x}$-rays from YSZ phase must traverse many microns of Ni or YSZ phases before reaching the detector detected, giving rise to significant absorption effect.

23. Faes, A., Hessler-Wyser, A., Presvytes, D., Vayenas, C. G. \& Van Herle, J. NickelZirconia Anode Degradation and Triple Phase Boundary Quantification from Microstructural Analysis. Fuel Cells 9, 841-851 (2009).

24. Suzuki, T. et al. Impact of Anode Microstructure on Solid Oxide Fuel Cells. Science 325, 852-855 (2009).

25. Mimura, H. et al. Breaking the $10 \mathrm{~nm}$ barrier in hard-X-ray focusing. Nat. Phys. 6 , $122-125(2010)$ 
26. Lombardo, J. J., Ristau, R. A., Harris, W. M. \& Chiu, W. K. S. Focused ion beam preparation of samples for X-ray nanotomography. Journal of Synchrotron Radiation 19, 789-796 (2012).

27. Giannuzzi, L. A. \& Stevie, F. A. A review of focused ion beam milling techniques for TEM specimen preparation. Micron 30, 197-204 (1999).

28. Li, J., Malis, T. \& Dionne, S. Recent advances in FIB-TEM specimen preparation techniques. Mater. Charact. 57, 64-70 (2006).

29. The build-in nonlinear fitting function in MatLab was used to find the best estimates.

30. Menzel, A. et al. Scanning transmission X-ray microscopy with a fast, framing pixel detector. Ultramicroscopy 110, 1143-1147 (2010).

\section{Acknowledgments}

The authors thank R. Conley, N. Bouet, and J Zhou for producing MLL optics for this work. The authors thank P. Fuesz, M. Holt and V. Rose for their support during the experiment. The authors also thank K. Lauer for help in control and A. Miceli for assistance in the detector setup. H. Y. and Y. S. C. thank M. de Jong and X. Huang for fruitful discussion on DPC algorithms. H. Y. thanks M. Lu for supplying the test pattern. Work at Brookhaven was supported by the Department of Energy, Office of Basic Energy Sciences under contract DE-AC-02-98CH10886. Work at Argonne, including use of the Advanced Photon Source and Center for Nanoscale Materials, was supported by the Department of Energy, Office of Basic Energy Sciences under contract DE-AC-02-06CH11357. H. C. K. would like to acknowledge the support by Basic Science Research Program through the National
Research Foundation of Korea (NRF) funded by the Ministry of Education, Science and Technology (MEST No. R15-2008-006-01000-0 and 2010-0023604). J.J.L. and W.K.S.C. acknowledge financial support from an Energy Frontier Research Center on Science Based Nano-Structure Design and Synthesis of Heterogeneous Functional Materials for Energy Systems (HeteroFoaM Center) funded by the U.S. Department of Energy, Office of Science, Office of Basic Energy Sciences (Award DE-SC0001061).

\section{Author contributions}

H.Y. and Y.S.C. planned and designed the experiment. H.Y. and Y.S.C. did the data analysis. H.Y., Y.S.C., J.M., E.N., J.K. and H.C.K. conducted the experiment. J.L.L. and W.K.S.C. prepared the FIB-produced SOFC sample and obtained SEM images. All authors contributed to the writing of the manuscript.

\section{Additional information}

Supplementary information accompanies this paper at http://www.nature.com/ scientificreports

Competing financial interests: The authors declare no competing financial interests. License: This work is licensed under a Creative Commons Attribution-NonCommercial-NoDerivs 3.0 Unported License. To view a copy of this license, visit http://creativecommons.org/licenses/by-nc-nd/3.0/

How to cite this article: Yan, H. et al. Quantitative $\mathrm{x}$-ray phase imaging at the nanoscale by multilayer Laue lenses. Sci. Rep. 3, 1307; DOI:10.1038/srep01307 (2013). 